Pacific Journal of Mathematics

INVARIANT MEANS AND THE STONE-ČECH

Carroll O. Wilde and KLAuS G. Wi 


\section{INVARIANT MEANS AND THE STONE-ČECH COMPACTIFICATION}

\section{Carroll Wilde and Klaus Witz}

In the first part of this paper the Arens multiplication on a space of bounded functions is used to simplify and extend results by Day and Frey on amenability of subsemigroups and ideals of a semigroup. For example it is shown that if $S$ is a left amenable cancellation semigroup then a subsemigroup $A$ of $S$ is left amenable if and only if each two right ideals of $A$ intersect. The remainder and major portion of this paper is devoted to relations between left invariant means on $m(S)$ and left ideals of $\beta S(=$ the Stone-Čch compactification of $S)$. We find: If $\mu$ is a left invariant mean on $m(S)$ and if $S$ has left cancellation then $\mathscr{S}(\mu)$, the support of $\mu$ considered as a Borel measure on $\beta(S)$, is a left ideal of $\beta(S)$. An application is that if $S$ is a left amenable semigroup and $I$ is a left ideal of $\beta S$, then $K(I)$, the $w^{*}$-closed convex hull of $I$, contains an extreme left invariant mean; if in addition $S$ has cancellation then $K(I)$ contains a left invariant mean which is the $w^{*}$-limit of a net of unweighted finite averages.

2. Preliminaries. For standard notation and terminology we follow Day [2] in functional analysis and Kelley [6] in topology. Specific terms and notation in amenable semigroups follow Day [1].

Let $S$ be any set, and let $m(S)$ be the Banach space of all bounded, real-valued functions on $S$, equipped with the supremum norm. A mean on $m(S)$ is a positive linear functional on $m(S)$ which has norm one; every mean $\mu$ satisfies $\mu(e)=1$, where $e$ is the function which is identically one on $S$. We denote by $M(=M(S))$ the set of all means on $m(S)$. Then $M$ is a nonempty, convex subset of $m(S)^{*}$; it is also compact in the $w^{*}$-topology, the only topology we consider in $m(S)^{*}$.

For each $s \in S, q(s)$ denotes the evaluation functional at $s$ :

$$
q s(x)=x(s)(x \in m(S)) \text {. }
$$

We have $q s \in M(s \in S)$ and $\beta S$, the Stone-Cěch compactification of the discrete space $S$, coincides with the $\left(w^{*}\right)$ closure of $q S$ in $m(S)^{*}$, so that $\beta S \leqq M$. The symbols $k(T), K(T)$ will always indicate the convex hull, resp. the $\left(w^{*}\right)$ closed convex hull, of any subset $T$ of $m(S)^{*}$; in particular, we write $k A$ for $k(q A)$ and $K A$ for $K(q A)$ when $A \leqq S$. Then we have $M=K S=K(\beta S)$.

Now suppose $S$ is a semigroup. Then each $s \in S$ determines two mappings, $l_{s}$ and $r_{s}$, on $m(S)$ defined by $l_{s} x(t)=x(s t)$ and $r_{s} x(t)=$ $x(t s)(t \in S, x \in m(S))$. A mean $\mu$ on $m(S)$ is left [right] invariant if 
and only if

$$
\mu\left(l_{s} x\right)=\mu(x)\left[\mu\left(r_{s} x\right)=\mu(x)\right]
$$

for all $s \in S$ and $x \in m(S)$; $\mu$ is invariant if and only if it is both left and right invariant. A semigroup $S$ is (left) [right] amenable if and only if there exists a (left) [right] invariant mean on $m(S)$. We denote by $L(=L(S))$ the set of all left invariant means on $m(S)$; then $L$ is a (possibly empty) closed convex subset of $M$.

Following Day [1], we define the Arens product, $\mu \odot \nu$, of two functionals $\mu, \nu \in m(S)^{*}$ by $(\mu \odot \nu) x=\mu(y)$, where $y \in m(S)$ is defined by $y(s)=\nu\left(l_{s} x\right)(s \in S)$. Arens' multiplication makes $m(S)^{*}$ into a semigroup and has the following properties: For fixed $\nu \in M$ and $\theta \in k(S)$ the mappings $\mu \rightarrow \mu \odot \nu$ and $\mu \rightarrow \theta \odot \mu$ are linear and $\left(w^{*}-w^{*}\right)$ continuous on $M$ (if $\theta \in M$ the mapping $\mu \rightarrow \theta \odot \mu$ may not be continuous), and the mapping $q$ is an isomorphism of the semigroup $S$ onto the semigroup $q S[1]$. It follows easily that $\beta S$ is a semigroup under $\odot, A^{-}$is a subsemigroup of $\beta S$ if $A$ is a subsemigroup of $S$, and the closure of a (left) [right] ideal of $S$ is a (left) [right] ideal of $\beta S$. Moreover $M$ is a semigroup under $\odot, K A$ is a subsemigroup of $M$ if $A$ is a subsemigroup of $S$, and if $I$ is a (left) [right] ideal of $S$, then $K I$ is a (left) [right] ideal of $M$. Finally if $I$ is a left ideal of $\beta S$ then $K I$ is a left ideal of $M$. For if $I$ is a left ideal of $\beta S$, then $\beta S \odot I \subseteq I$ and in particular, $q S \odot I \subseteq I$. Hence $k S \odot k I \subseteq k I$, therefore

$$
k S \odot K I \sqsubseteq K I,
$$

and therefore $M \odot K I \subseteq K I$ by $\left(w^{*}-w^{*}\right)$ continuity of Arens' multiplication in the second, resp. first, variable.

The significance of the Arens multiplication for left invariance of means is this: For any semigroup $S, \mu \in L$ if and only if $\nu \odot \mu=\mu$ for all $\nu \in M[1$, p. 530]. (This is clearly equivalent to the condition $q s \odot \mu=\mu(s \in S))$. In general, define the kernel of the semigroup $M, \operatorname{Ker}(M)$, to be the smallest closed two-sided ideal of $M$. We always have $\operatorname{Ker}(M) \neq \varphi$ (by compactness of $M$ ), and $L \neq \varphi$ if and only if $\operatorname{Ker}(M)$ is the smallest closed right ideal of $M$, in which case $L=\operatorname{Ker}(M)[10]$.

The ideas of the preceding two paragraphs are used repeatedly throughout this paper.

3. A theorem of Day and some applications. We first extend the theorem of Day mentioned in the introduction.

THEOREM 3.1. If $S$ is a semigroup and $A$ is a subsemigroup of $S$, let $L_{A}=\{\mu \in M(S): q s \odot \mu=\mu$ for all $s \in A\}$, and let $N_{A}=L_{A} \cap K A$. 
Then the following conditions are equivalent:

(i) $A$ is left amenable.

(ii) There exists $\mu \in L_{A}$ such that $\mu\left(X_{A}\right)>0\left(X_{A}\right.$ is the characteristic function of $A$ ).

(iii) $N_{A} \neq \varphi$.

Proof. (ii) $\Rightarrow$ (i). This is Day's Theorem with the observation that his proof requires invariance of $\mu$ over $A$ only.

(iii) $\Rightarrow$ (ii). Since $\mu \in K A$, we have $\mu\left(X_{A}\right)=1$.

(i) $\Rightarrow$ (iii). Let $\nu$ be a left invariant mean on $m(A)$ and define $\mu=\Pi^{*} \nu$, where $\Pi$ is the restriction map of $m(S)$ onto $m(A)$ defined by $\Pi x(t)=x(t)$ for $t \in A, x \in m(S)$ [see 1, p. 512]. It is easily checked that $\mu$ is a mean on $m(S)$. Now $\Pi X_{A}$ is the function identically one on $A$, so $\mu\left(X_{A}\right)=\nu\left(\Pi X_{A}\right)=1$. Therefore $\mu \in K A$. For we can write

$$
\mu=\lambda \mu_{1}+(1-\lambda) \mu_{2},
$$

where $0 \leqq \lambda \leqq 1, \mu_{1} \in K A$, and $\mu_{2} \in K A^{\prime}\left(A^{\prime}=S \backslash A\right)$. Evaluating both sides at $X_{A}$ yields $\lambda=1$, whence $\mu=\mu_{1} \in K A$. It remains to show that $\mu \in L_{\Delta}$. If $s \in A$ and $x \in m(S)$ then

$$
(q s \odot \mu) x=q s(y),
$$

where $y(t)=\mu\left(l_{t} x\right)$ and therefore

$$
(q s \odot \mu) x=y(s)=\mu\left(l_{s} x\right)=\nu\left(\Pi\left(l_{s} x\right)\right)=\nu\left(\lambda_{s}(\Pi x)\right)=\nu(\Pi x)=\mu(x)
$$

(here $l_{s}$ is the left shift by $s$ in $m(A)$ ).

Theorem 3.1 and the ideas involved in its proof combine with facts mentioned at the end of $\S 2$ to give quite short proofs for some known results and to extend others. For example, the following corollary is due to E. Granirer (unpublished); his proof is independent of ours.

Corollary 3.2. Suppose $G$ is any group and $A$ is a subsemigroup of $G$ which generates $G$. Then $A$ is left amenable if and only if $K A \cap L(G) \neq \varphi$.

Proof. Only the forward implication is not apparent by Day's Theorem itself. By Theorem 3.1, there exists $\mu \in K A$ such that

$$
q g \odot \mu=\mu
$$

for all $g \in A$. Thus if $g \in A$, then

$$
q g^{-1} \odot \mu=q g^{-1} \odot(q g \odot \mu)=\left(q g \odot q g^{-1}\right) \odot \mu=q u \odot \mu=\mu,
$$

where $u$ is the group identity. Since each $g \in G$ can be written as a 
product of elements which are in $A$ or $A^{-1}$, we are finished.

Corollary 3.3. (i) If a semigroup $S$ contains a left amenable left ideal $I$, then $S$ is left amenable.

(ii) Conversely, if $S$ is a left amenable semigroup, then each left or right ideal $I$ of $S$ is left amenable.

Proof. Part (i) appears in Frey [4]; Frey also proves (ii) for right ideals but states that he was unable to obtain the result for left ideals. In our setting everything becomes quite simple. For (i), we note that since $I$ is left amenable, there exists $\mu \in K I$ such that $q t \odot \mu=\mu$ for all $t \in I$ by Theorem 3.1. If $s \in S$, choose any $t \in I$; then $q s \odot \mu=q s \odot(q t \odot \mu)=q s t \odot \mu=\mu$; hence $\mu \in L(S)$. For (ii), suppose $S$ is left amenable and let $I$ be a left [right] ideal of $S$. Then $K I$ is a left [right] ideal of $M(S)$ and must intersect the two-sided ideal $L(S)=\operatorname{Ker}(M)$. That is, there exists $\mu \in L$ such that $\mu\left(X_{I}\right)=1$, and the result follows from Day's Theorem.

We now use Theorem 3.1 to sharpen another result of Frey, namely: In a left amenable, two-sided cancellation semigroup, every subsemigroup is left amenable if and only if every subsemigroup satisfies the condition that its family of all right ideals has the finite intersection property. We show (Theorem 3.5) that this last condition characterizes any left amenable subsemigroup.

Lemma 3.4. Let $G$ be a group, and let $A$ be a subsemigroup of $G$ such that $A$ generates $G$ and the family of all right ideals of $A$ has the finite intersection property. If $g \in G$, then $g A \cap A$ is a right ideal of $A$.

Proof. We need only prove that $g A \cap A \neq \varphi$ for an arbitrary $g \in G$. This is clear if $g \in A$ or $g^{-1} \in A$. If $g \in G$, we can write

$$
g=g_{1} g_{2} \cdots g_{n},
$$

where $g_{i} \in A$ or $A^{-1}$ for $1 \leqq i \leqq n$. Letting $h=g_{.} g_{3} \cdots g_{n}$, we have $g A \cap A=g_{1} h A \cap A$, and by induction, $h A \cap A \neq \varphi$. If $g_{1} \in A$, then $g_{1}(h A \cap A) \neq \varphi$, and

$$
g_{1}(h A \cap A)=\left(g_{1}(h A \cap A)\right) \cap A \cong g_{1} h A \cap A=g A \cap A,
$$

so $g A \cap A \neq \varnothing$ in this case. If $g_{1} \in A^{-1}$, then

$$
(h A \cap A) \cap g_{1}^{-1} A \neq \varnothing
$$

because it is the intersection of two right ideals of $A$. Hence

$$
h A \cap g_{1}^{-1} A \neq \varnothing
$$


and so $g A \cap A=g_{1}\left(h A \cap g_{1}^{-1} A\right) \neq \varphi$.

THEOREM 3.5. Let $S$ be a left amenable, two-sided cancellation semigroup. Then the following conditions on a subsemigroup $A$ of $S$ are equivalent:

(i) $A$ is left amenable.

(ii) The family of all right ideals of $A$ has the finite intersection property.

Proof. Frey shows that condition (ii) is necessary for left amenability of any semigroup; we give a shorter proof. Let $T$ be any left amenable semigroup and assume $I$ and $J$ are two disjoint right ideals of $T$. Then $K I \cap K J=\varphi$ in $M(T)$; for if $\mu \in K I \cap K J$, then $\mu\left(X_{I}\right)=\mu\left(X_{J}\right)=1$, which would imply $\mu(e) \geqq 2$. Hence $M(T)$ contains two disjoint closed right ideals, contradicting that $L(T)=\operatorname{Ker}(M(T))$ is the smallest closed right ideal of $M(T)$. Taking $T=A$, we have (i) $\Rightarrow$ (ii). To prove (ii) $\Rightarrow$ (i), we use the following theorem of Ore [cf. 7, p. 392]: A two-sided cancellation semigroup $T$ is embeddable in a group if, given $s, t \in T$, there exist $s^{\prime}, t^{\prime} \in T$ such that $s s^{\prime}=t t^{\prime}$. This tells us that $S$ is embeddable in a group $G$, and we can assume that $S$ generates $G$. By Corollary 3.2, $G$ is amenable. By Lemma 3.4, $\{g A \cap A: g \in G\}$ has the finite intersection property; hence so does $\left\{(g A \cap A)^{-}: g \in G\right\}$ in $\beta G$. Set $E=\cap\left\{(g A \cap A)^{-}: \mathrm{g} \in G\right\}$. By compactness, $E \neq \varnothing$, and clearly $E \subseteq A^{-}$and $E=\cap\left\{(g A)^{-:}: g \in G\right\}$. We shall show that $E$ is a left ideal of $\beta G$. Let $h \in G$; since

$$
q(h A)^{-}=q h \odot A^{-},
$$

we have $q h \odot E \subseteq \cap\left\{(h g A)^{-}: g \in G\right\}$. But

$$
\cap\left\{(h g A)^{-}: g \in G\right\}=\cap\left\{(g A)^{-}: g \in G\right\}=E
$$

because $G$ is a group and so the intersections are over the same class of sets. Hence $q h \odot E \subseteq E$. It follows that $q G \odot E \subseteq E$ and

$$
\beta G \odot E \subseteq E .
$$

Thus $A^{-}$contains a left ideal of $\beta G$ (namely $E$ ), and so $K\left(A^{-}\right)=K A$ contains a left ideal of $M(G)$ (namely $K E$ ). The proof of Corollary 3.3 , part (ii), shows that $A$ is left amenable.

We note the following immediate corollary to the proof of Theorem 3.5 .

Corollary 3.6. A left amenable, two-sided cancellation semigroup is embeddable in an amenable group. 
4. Supports. We identify $m(S)$ with $C(\beta S)$ as usual and regard $M$ as a subset of $C(\beta S)^{*}$. Thus for each $\mu \in M$ there is a unique regular Borel measure $\bar{\mu}$ on $\beta S$ which satisfies

$$
\mu(x)=\int_{\beta, S} \bar{x} d \bar{\mu}
$$

(where $\bar{x}$ is the unique continuous extension of $x$ from $S$ to $\beta S$ ), and $\bar{\mu}(\beta S)=1$. Let $\delta(\mu)$ be the support of $\bar{\mu}$, i.e., the smallest closed subset $F$ of $\beta S$ such that $\mu(F)=1$.

Lemma 4.1. If $\mu \in M$ then

$$
\delta(\mu)=\cap\{\bar{A}: A \subseteq S \text { and } \mu \in K A\} .
$$

Proof. Denote the intersection on the right by $F_{0}$. It is well known ${ }^{1}$ that if $F$ is a closed subset of $\beta S$ and if $\mu \in M$ then

$$
\bar{\mu}(F)=1 \text { (i.e., } \delta(\mu) \subset F \text { ) if and only if } \mu \in K F \text {. }
$$

Hence $\delta(\mu) \subset F_{0}$, and since $S$ is dense in $\beta S, F_{0} \subset \delta(\mu)$.

Recall that a subset $A$ of a compact convex subset $K$ of a locally convex space is extremal in $K$ if and only if $A$ is compact, convex, and every open segment in $K$ which contains a point of $A$ lies wholly in $A$ [2, p. 78].

Lemma 4.2. (i) If $D$ is a closed subset of $\beta S$, then $D=\mathscr{L}(K D)$. If $E$ is an extremal subset of $M$, then $E=K(\mathscr{L}(E)$ ).

(ii) The mapping $D \rightarrow K D$ is a one-to-one correspondence between the class of all closed subsets $D$ of $\beta S$ and the class of all extremal subsets of $M$.

Proof. (i) By Lemma 4.1, $\mathscr{S}(K D) \subseteq D$, and a separation argument shows that the inclusion is not proper. Let $E$ be an extremal subset of $M$. Then clearly $E \subseteq K(\mathscr{S} E)$ and by the KreinMil'man Theorem, $E$ is the closed convex hull of its set of extreme points. Since $E$ is an extremal set in $M$, each extreme point of $E$ is an extreme point of $M$ and hence in $\beta S$. Hence $E=K D$, where $D$ is a subset of $\beta S$, and $D$ can be assumed closed. Thus

$$
K(\mathscr{S} E) \subseteq K D=E .
$$

(ii) The mapping $D \rightarrow K D$ on the closed subsets $D$ of $\beta S$ is oneto-one, again by a separation argument. To see that $K D$ is an extremal subset of $M$, let $\mu \in K D$ and suppose $\mu=\lambda \mu_{1}+(1-\lambda) \mu_{2}$ with

1 See, e.g., Bourbaki's Integration, Chapter III, p. 87. 
$0<\lambda<1$ and $\mu_{1}, \mu_{2} \in M$. Fix $A \cong S$ such that $K A \supseteqq K D$; then $\mu \in K A$, so that $\mu\left(X_{A}\right)=1$. Since $\mu_{1}, \mu_{2} \in M$, we have

$$
\mu_{1}\left(X_{A}\right), \mu_{2}\left(X_{A}\right) \leqq 1,
$$

and if either is less than one, we would have

$$
1=\mu\left(X_{A}\right)=\lambda \mu_{1}\left(X_{A}\right)+(1-\lambda) \mu_{2}\left(X_{A}\right)<\lambda \cdot 1+(1-\lambda) \cdot 1=1 .
$$

Hence $\mu_{1}\left(X_{A}\right)=\mu_{2}\left(X_{A}\right)=1$, so that $\mu_{1}, \mu_{2} \in K A$. Since $A \subseteq S$ was arbitrary subject only to the restriction $K A \supseteqq K D$, we have

$$
\begin{aligned}
\mu_{1}, \mu_{2} \in \cap\{K A: A & \leqq S \text { and } K A \supseteqq K D\} \\
& =K\left(\cap\left\{A^{-}: A \cong S \text { and } K A \supseteqq K D\right\}\right. \\
& =K(\mathscr{S}(K D))=K D .
\end{aligned}
$$

We now assume that $S$ is a semigroup and apply the preceding results. Namely, we show that for certain semigroups the supporting sets of invariant means are left ideals of $\beta S$. The general case is still open (see the comment after 5.1.)

THEOREM 4.3. Let $S$ be a left amenable, two-sided cancellation semigroup. If $L_{0} \subseteq L, L_{0} \neq \varphi$, then $\mathscr{S}\left(L_{0}\right)$ is a closed left ideal of $\beta S$.

Proof. Suppose first that $S=G$, a group. Let $\omega \in \mathscr{S}\left(L_{0}\right)$ and let $g \in G$. Then $q g \odot \omega \in q g \odot\left(A^{-}\right)=(g A)^{-}$for all $A \subseteq G$ such that $K A \supseteqq L_{0}$. Thus $q g \odot \omega \in \cap\left\{(g A)^{-}: A \subseteq G\right.$ and $\left.K A \supseteqq L_{0}\right\}$. Now $L_{0} \leqq K A$ if and only if $L_{0} \subseteq K(h A)$ for each $h \in G$; for if $\mu \in L_{0} \leqq K A$, then $\mu=q h \odot \mu \in q h \odot K A=K(h A)$, and if $\mu \in X K(h A)$, then $\mu=q h \odot \nu$ with $\nu \in K A$, so that $\mu=q h^{-1} \odot \mu=q h^{-1} \odot(q h \odot \nu)=\nu \in K A$. Hence $\left\{A: A \subseteq G\right.$ and $\left.K A \supseteqq L_{0}\right\}=\left\{g A: A \cong G\right.$ and $\left.K A \supseteqq L_{0}\right\}$ so that

$$
q g \odot \omega \in \mathscr{S}\left(L_{0}\right) .
$$

Then $q G \odot \omega \subseteq \mathscr{S}\left(L_{0}\right), \beta G \odot \omega \subseteq \mathscr{S}\left(L_{0}\right)$, and $\beta \mathrm{G} \odot \mathscr{S}\left(L_{0}\right) \subseteq \mathscr{S}\left(L_{0}\right)$. To remove the group restriction, we embed $S$ in a group $G$ such that $S$ generates $G$. The mapping $\Pi^{*}$ identifies $\beta S$ with $S^{-}$in $\beta G$, and by Corollary $2.2, \Pi^{*}\left(L_{0}\right)$ is a subset of the left invariant means on $m(G)$. Applying the result for groups, we get that $\left(\Pi^{*} L_{0}\right)$ is a closed left ideal of $S^{-}$. Taking the inverse under $\Pi^{*}$ yields the desired result.

In particular, if $\mu$ is a left invariant mean on $m(S)$, then $\mathscr{S}(\mu)$ is a left ideal of $\beta S$ (provided cancellation is present).

THEOREM 4.4. If $S$ is a left amenable semigroup and $I$ is a left ideal of $\beta S$, then $K I$ contains an extreme point of $L$. 
Proof. Since $I$ is a left ideal of $\beta S, K I$ is a left ideal of $M$, so $K I \cap L \neq \varphi$, and since $K I$ is an extremal subset of $M$ and $L$ is compact and convex, we have that $K I \cap L$ is an extremal subset of $L$. Hence $K I \cap L$ contains an extreme point of $L[2, \mathrm{p} .78]$.

In $\beta S$ minimal left ideals exist, are closed, are pairwise disjoint, and are usually abundant. Then Theorem 4.4 and the facts noted in the proof of 4.1 imply that there are at least as many extreme points of $L$ as there are minimal left ideals of $\beta S$. In the special case where $S=N$, the additive semigroup of positive integers, Raimi [9] recently showed that there are at least two extreme points of $L$ in $K I$ for each minimal left ideal $I$ of $\beta N$. We remark that if $I$ is a minimal left ideal of $\beta S$, then $I=\mathscr{S}\left(L_{0}\right)$ for each subset $L_{0}$ of $K I \cap L$.

We close this section with a corollary to Theorem 4.3.

COROLlary 4.5. If $S$ is a left amenable, two-sided cancellation semigroup and $A$ is a subsemigroup of $S$ which is not left amenable, then $\left(A^{\prime}\right)^{-}$contains a (minimal) left ideal of $\beta S$.

Proof. By Day's Theorem, $\mu\left(X_{\Delta}\right)=0$ for all $\mu \in L$; thus $L \subseteq K A^{\prime}$, and so $\mathscr{S}(L) \subseteq\left(A^{\prime}\right)^{-}$.

5. On a theorem of Mitchell. In his thesis [8, p. 39], T. Mitchell proved the following theorem:

(*) Let $S$ be a left amenable semigroup, and let $A \subseteq S$. Then there exists $\mu \in L$ such that $\mu\left(X_{A}\right)=1$ (i.e., $\mu \in K A$ ) if and only if given any finite subset $F$ of $S$, there exists $s \in S$ such that $F s \subseteq A$.

We give an easy and different proof of a related fact.

LEMMA 5.1. If $S$ is any semigroup, then the following conditions on $A \subseteq S$ are equivalent:

(i) $A^{-}$contains a (closed) left ideal of $\beta S$.

(ii) For each finite subset $F$ of $S$ there exists $s \in S$ such that $F s \subseteq A$.

Proof. (i) $\Rightarrow$ (ii). Let $I \subseteq A^{-}$be a left ideal of $\beta S$. Let $\omega \in I$ and take a net $\left\{s_{n} ; n \in N\right\}$ such that $q s_{n} \rightarrow \omega$. If $F$ is a finite subset of $S, F=\left\{t_{1}, t_{2}, \cdots, t_{k}\right\}$, then $q\left(t_{i} s_{n}\right)=q t_{i} \odot q s_{n} \rightarrow q t_{i} \odot \omega \in I$ for $1 \leqq i \leqq k$; and since $A^{-}$is an open set containing $I$, there exists $n_{0} \in N$ such that $n \geqq n_{0}$ implies $q t_{i} \odot q s_{n} \in A^{-}$for $1 \leqq i \leqq k$; in particular,

$$
F s_{n_{0}} \subseteq A .
$$

(ii) $\Rightarrow$ (i). Let $\mathscr{F}$ be the family of all finite subsets of $S$ directed upward by inclusion. Define a net over $\mathscr{F}$ as follows: For each 
$F \in \mathscr{F}$ choose an $s_{F}$ such that $F s_{F^{\prime}} \subseteq A$. By compactness, there is a subnet $\left\{s_{n} ; n \in N\right\}$ of the net $\left\{s_{F} ; F \in \mathscr{F}\right\}$ and $\omega \in \beta S$ such that $q s_{n} \rightarrow \omega$. Fix $\mathrm{s} \in S$, and put $F_{0}=\{s\}$. Then there exists $n_{0} \in N$ such that $n \geqq n_{0}$ implies that $s_{n}=s_{F^{\prime}}$ with $F \geqq F_{0}$. Since

$$
s s_{n} \in F_{0} s_{n} \subseteq F s_{n} \subseteq A
$$

for all $n \geqq n_{0}$ and $q s \odot q s_{n} \rightarrow q s \odot \omega$, we have $q s \odot \omega \in A^{-}$. Since $s \in S$ was arbitrary, we have $q S \odot \omega \subset A^{-}$, and so $\beta S \odot \omega \subseteq A^{-}$. Since $\beta S \odot \omega$ is a closed left ideal of $\beta S$, the lemma is proved.

Let $S$ be a left amenable semigroup, and let $A \subseteq S$. It follows as in Corollary 3.3 that if $A^{-}$contains a left ideal of $\beta S$, then

$$
K A \cap L \neq \varphi,
$$

and in view of Lemma 5.1, this is one half of $\left({ }^{*}\right)$. Conversely, if $A \subseteq S$ and $K A \cap L \neq \varphi$, then by (*) and Lemma 5.1, $A^{-}$must contain a left ideal of $\beta S$. Observe that we get the same conclusion independent of $\left({ }^{*}\right)$ from Theorem 4.3 in case two-sided cancellation holds in $S$, and this suggests that it may be possible to extend Theorem 4.3 to more general semigroups.

For our next and final result we need Frey's generalization [4] of Folner's Theorem [3]: A two-sided cancellation semigroup $S$ is left amenable if and only if for each $\varepsilon \in(0,1)$ and each finite subset $F$ of $S$ there exists a finite subset $E$ of $S$ such that $(|s E \cap E| /|E|) \geqq 1-\varepsilon$ for each $s \in F$ (here $|D|$ denotes the cardinality of $D \subseteq S$ ).

Definition. Let $S$ be a semigroup. An arithmetic average on $S$ is a mean on $m(S)$ of the form $\mu_{E}=1 /|E| \sum_{s \epsilon_{E}} q s$, where $E$ is a finite subset of $S$.

THEOREM 5.2. Let $S$ be a left amenable, two-sided cancellation semigroup and $L_{0} \subseteq L$. Then there exists $\mu \in K\left(\mathscr{S} L_{0}\right) \cap L$ such that $\mu$ is the limit of a net of arithmetic averages on $S$.

Proof. We remark that our proof assumes that $S$ is infinite; the theorem is trivial otherwise. We consider three directed sets, $\mathscr{F}, \mathscr{I}, \mathscr{A}: \mathscr{F}$ is the system of finite subsets of $S$ directed upward by inclusion; $\mathscr{I}$ is the real interval $(0,1)$ directed upward by $\leqq ; \mathscr{K}$ is the system of all subsets $A$ of $S$ such that $K A \supseteqq L_{0}$, directed downward by inclusion. Let $(\mathscr{F}, \mathscr{F}, \mathscr{N})$ be the product directed system and fix $n=(F, \varepsilon, A) \in(\mathscr{F}, \mathscr{F}, \mathscr{O})$. By the Frey-Folner theorem, there exists a finite subset $E_{F, \varepsilon}$ of $S$ such that $\left(\left|s E_{F, \varepsilon} \cap E_{F, \varepsilon}\right| /\left|E_{F^{\prime}, \varepsilon}\right|\right) \geqq 1-\varepsilon$ for each $s \in F$. By Lemma 5.1, there exists $s_{F, s, A}=s_{n} \in S$ such that $E_{F^{\prime},} s_{n} \subseteq A$. Let $D_{n}=E_{F^{\prime}, s} s_{n}$, and let $\mu_{n}$ be the arithmetic mean over $D_{n}$. Since $n \in N=(\mathscr{F}, \mathscr{I}, \mathscr{C})$ was arbitrary, $\left\{\mu_{n} ; n \in N\right\}$ is a net 
over N. By cancellation,

$$
\begin{aligned}
\left(\left|s D_{n} \cap D_{n}\right| /\left|D_{n}\right|\right) & =\left(\left|s\left(E_{F^{\prime}, \varepsilon} s_{n}\right) \cap\left(E_{F^{\prime}, \varepsilon} s_{n}\right)\right| /\left|E_{F^{\prime}, \varepsilon} s_{n}\right|\right) \\
& =\left(\left|s E_{F^{\prime}, \varepsilon} \cap E_{F^{\prime}, \varepsilon}^{\prime}\right| /\left|E_{F^{\prime}, \varepsilon}\right|\right) \geqq 1-\varepsilon
\end{aligned}
$$

for each $s \in F$. If $t \in S$ and $x \in m(S)$, then

$$
\begin{aligned}
\left|\left(q t \odot \mu_{n}\right)(x)-\mu_{n}(x)\right| & =\left.|| D_{n}\right|^{-1} \sum_{s \in D_{n}}(x(t s)-x(s)) \mid \\
& \leqq\left|D_{n}\right|^{-1} \cdot \varepsilon \cdot 2\|x\| \cdot\left|D_{n}\right| \rightarrow 0 ;
\end{aligned}
$$

hence $\left\{\mu_{n}\right\}$ converges to left invariance [1, p. 520]. Let $\mu$ be a cluster point of $\left\{\mu_{n} ; n \in N\right\}$, so that $\mu \in L\left[1\right.$, p. 520]. Fix $A_{0} \in \mathscr{A}$. If $A \geqq A_{0}$ and $F \in \mathscr{F}, \varepsilon \in \mathscr{J}$ are arbitrary, then $D_{n}=E_{F^{\prime}, \varepsilon} s_{n} \subseteq A \subseteq A_{0}$, so that $\mu_{n} \in K A_{0}$. This implies that $\mu \in K A_{0}$; and since $A_{0}$ was arbitrary in $\mathscr{\mathscr { C }}$, we have $\mu \in K\left(\mathscr{S} L_{0}\right)$.

In particular, if $I$ is a minimal left ideal of $\beta S$, then $K I$ contains at least one left invariant mean $\mu_{I}$ which is the $\left(w^{*-}\right)$ limit of a net of arithmetic averages. Thus there are at least as many invariant means of this type as there are distinct minimal left ideals in $\beta S$ (see the discussion after Theorem 4.6).

\section{REFERENCES}

1. M. M. Day, Amenable semigroups, Illinois J. Math. 1, (1957), 509-544.

2. — Normed Linear Spaces, second printing corrected, New York, Academic Press, Inc., 1962.

3. E. Folner, On groups with full Banach mean values, Math. Scand. 3, (1955), 243254.

4. A. H. Frey, Studies on amenable semigroups, Thesis, University of Washington, Seattle, 1960.

5. M. Jerison, A property of extreme points of compact convex sets, Proc. Amer. Math. Soc. 5 (1954), 782-783.

6. J. L. Kelley, General Topology, New York, Van Nostrand, 1955.

7. E. S. Ljapin, Semigroups, Amer, Math. Soc. Translations of Math. Monographs

3.

8. T. Mitchell, Invariant means on semigroups and the constant functions, Thesis, Illinois Institute of Technology, Chicago, 1964.

9. R. A. Raimi, Minimal sets and ergodic measures in $\beta N-N$, Bull. Amer. Math. Soc. 70 (1964), 711-712.

10. K. G. Witz, Applications of a compactification for bounded operator semigroups, Illinois J. Math. 8 (1964), 685-696.

Received May 10, 1965. Supported by the Danforth Foundation and the National Science Foundation. Some portions of this paper form part of the first author's doctoral dissertation prepared under the direction of M. M. Day. The authors are grateful to the referee for suggesting a number of improvements.

UNIVERSITY OF ILLINOIS AND

UNIVERSITY OF MINNESOTA 


\section{PACIFIC JOURNAL OF MATHEMATICS}

\section{EDITORS}

\section{H. SAMELSON}

Stanford University

Stanford, California

\author{
J. P. JANS \\ University of Washington \\ Seattle, Washington 98105
}

\section{J. DuGUNDJI}

University of Southern California

Los Angeles, California 90007

RichaRd ARENS

University of California

Los Angeles, California 90024

\section{ASSOCIATE EDITORS}

E. F. BECKENBACH
B. H. NeUManN

F. WOLF

K. YOSIDA

\section{SUPPORTING INSTITUTIONS}

UNIVERSITY OF BRITISH COLUMBIA

CALIFORNIA INSTITUTE OF TECHNOLOGY

UNIVERSITY OF CALIFORNIA

MONTANA STATE UNIVERSITY

UNIVERSITY OF NEVADA

NEW MEXICO STATE UNIVERSITY

OREGON STATE UNIVERSITY

UNIVERSITY OF OREGON

OSAKA UNIVERSITY

UNIVERSITY OF SOUTHERN CALIFORNIA
STANFORD UNIVERSITY

UNIVERSITY OF TOKYO

UNIVERSITY OF UTAH

WASHINGTON STATE UNIVERSITY

UNIVERSITY OF WASHINGTON

AMERICAN MATHEMATICAL SOCIETY

CHEVRON RESEARCH CORPORATION

TRW SYSTEMS

NAVAL ORDNANCE TEST STATION

Mathematical papers intended for publication in the Pacific Journal of Mathematics should be typewritten (double spaced). The first paragraph or two must be capable of being used separately as a synopsis of the entire paper. It should not contain references to the bibliography. Manuscripts may be sent to any one of the four editors. All other communications to the editors should be addressed to the managing editor, Richard Arens at the University of California, Los Angeles, California 90024.

50 reprints per author of each article are furnished free of charge; additional copies may be obtained at cost in multiples of 50 .

The Pacific Journal of Mathematics is published monthly. Effective with Volume 16 the price per volume ( 3 numbers) is $\$ 8.00$; single issues, $\$ 3.00$. Special price for current issues to individual faculty members of supporting institutions and to individual members of the American Mathematical Society: $\$ 4.00$ per volume; single issues $\$ 1.50$. Back numbers are available.

Subscriptions, orders for back numbers, and changes of address should be sent to Pacific Journal of Mathematics, 103 Highland Boulevard, Berkeley 8, California.

Printed at Kokusai Bunken Insatsusha (International Academic Printing Co., Ltd.), No. 6, 2-chome, Fujimi-cho, Chiyoda-ku, Tokyo, Japan.

PUBLISHED BY PACIFIC JOURNAL OF MATHEMATICS, A NON-PROFIT CORPORATION

The Supporting Institutions listed above contribute to the cost of publication of this Journal, but they are not owners or publishers and have no responsibility for its content or policies. 


\section{Pacific Journal of Mathematics}

\section{Vol. 21, No. $3 \quad$ BadMonth, 1967}

Richard Allen Askey, A transplantation theorem for Jacobi coefficients . . . 393

Raymond Balbes, Projective and injective distributive lattices .......... 405

Raymond Balbes and Alfred Horn, Order sums of distributive lattices . . . . 421

Donald Charles Benson, Nonconstant locally recurrent functions ........ 437

Allen Richard Bernstein, Invariant subspaces of polynomially compact operators on Banach space ............................... 445

Robert F. Brown, Fixed points and fibre ................... 465

David Geoffrey Cantor, On the Stone-Weierstrass approximation theorem for valued fields ................................ 473

James Walton England, Stability in topological dynamics .............. 479

Alessandro Figà-Talamanca and Daniel Rider, A theorem on random

Fourier series on noncommutative groups.................. 487

Sav Roman Harasymiv, A note of dilations in $L^{p} \ldots \ldots \ldots \ldots \ldots \ldots . \ldots 493$

J. G. Kalbfleisch, A uniqueness theorem for edge-chromatic graphs ....... 503

Richard Paul Kelisky and Theodore Joseph Rivlin, Iterates of Bernstein polynomials .................................... 511

D. G. Larman, On the union of two starshaped sets ............... 521

Henry B. Mann, Josephine Mitchell and Lowell Schoenfeld, Properties of differential forms in $n$ real variables ...................... 525

John W. Moon and Leo Moser, Generating oriented graphs by means of team comparisons .

Veikko Nevanlinna, A refinement of Selberg's asymptotic equation ...

Ulrich Oberst, Relative satellites and derived functors of functors with additive domain ..............................

John Vincent Ryff, On Muirhead's theorem...............

Carroll O. Wilde and Klaus G. Witz, Invariant means and the Stone-Čech compactification 